\title{
Supervised continuous land cover change detection in a critically endangered shrubland ecosystem
}

\author{
Glenn R. Moncrieff ${ }^{1,2, \bowtie}$ \\ ${ }^{1}$ Fynbos Node, South African Environmental Observation Network, Private Bag X7, Rhodes Drive, Claremont 7735, South Africa \\ ${ }^{2}$ Centre for Statistics in Ecology, Environment and Conservation, Department of Statistical Sciences, University of Cape Town, Private Bag X3, Rondebosch 7701, South \\ Africa
}

\begin{abstract}
Existing efforts to rapidly detect land cover change in satellite 45 image time-series have mostly focused on forested ecosystems 46 in the tropics and northern hemisphere. The notable difference in reflectance that occurs following deforestation allow for unsupervised methods, often with manually determined thresholds, to detect land cover change with relative accuracy. Less progress has been made in detecting change in low productiv- 50 ity, disturbance-prone vegetation such as grasslands and shrub- 51 lands, where natural dynamics can be difficult to distinguish 52 from habitat loss. Renosterveld is a hyperdiverse, critically endangered shrubland ecosystem in South Africa with less than $5-10 \%$ of its original extent remaining in small, highly fragmented patches. I demonstrate that supervised classification of satellite image time series using neural networks can accurately detect the transformation of Renosterveld within a few days of its occurrence, and that trained models are suitable for ${ }^{5}$ operational continuous monitoring. A training dataset of pre- 59 cisely dated vegetation change events between 2016 and 202060 was obtained from daily, high resolution Planet labs satellite 61 data. This dataset was then used to train 1D convolutional 62 neural networks and Transformers to continuously classify land cover change events in multivariate time-series of vegetation activity from Sentinel 2 satellites as new data becomes available. These models reached a f-score of 0.93 , a $61 \%$ improvement over the f-score of 0.57 achieved using an unsupervised method designed for forested ecosystems. Models have been deployed to operational use and are producing updated detections of habitat loss every 10 days. There is great potential for supervised ${ }^{69}$ approaches to continuous monitoring of habitat loss in ecosys- 70 tems with complex natural dynamics. A key limiting step is the 71 development of accurately dated labelled datasets of land cover 72 change events with which to train machine learning classifiers.
\end{abstract}

Land cover change | Land cover monitoring | Deep learning | Renosterveld | 74 Threatened ecosystems | Sentinel 2 | Planet labs

Correspondence: glenn@saeon.ac.za

\section{Introduction}

Land cover change is the single largest cause underlying the global biodiversity crisis (Brondizio et al., 2019). It is esti- 81 mated that at least $21 \%$ of all terrestrial plant extinctions are 82 directly related to agriculture alone (Le Roux et al., 2019). A 83 core component of efforts to halt biodiversity loss and climate 84 change are remote sensing-based monitoring systems built 85 upon satellite-derived earth observation data (Turner, 2014; 86 Turner et al., 2003; Yang et al., 2013). These systems allow 87 up-to-date reporting on trends in habitat loss, and in some instances, near-real time alerts that may aid interventions and enforcement. Typically, temperate and tropical forests are the focal ecosystems for applications, examples of which include Global Forest Watch and Terra-i.

While forests are indeed globally important stores of carbon and home to myriad species, far less attention has been paid to developing remote sensing-based monitoring for use in open ecosystems where trees may be present but not dominant, such as savannas, grasslands, shrublands and deserts. Open ecosystems cover a large proportion of the globe, make up $40 \%$ of the global total ecosystem organic carbon and harbour a substantial proportion of the world's biological diversity (Bond, 2019; Poulter et al., 2014)). Failure to develop capacity for change detection in open ecosystems would exclude the majority of the earth's terrestrial surface from monitoring. Detecting abnormal change in forested ecosystems is relatively simple in comparison to open ecosystems. The spectral signature of a closed-canopy forest stands in stark contrast to the sparsely vegetated or bare ground that remains after deforestation. In contrast, the natural vegetation state in open ecosystems can vary dramatically due to natural disturbances, long-term trends or cyclical functions, such as those relating to fire, seasonality and interannual weather variations (Slingsby et al., 2020; Wilson et al., 2015). This variability found within healthy ecosystems can obscure spectral change that occurs when intact natural vegetation is converted to nonnatural land use.

The Cape Floristic region (CFR) in South Africa is home to some of the world's most floristically diverse open ecosystems (Manning and Goldblatt, 2012; Myers et al., 2000). Within the CFR the most threatened vegetation type is the grassy shrubland known as Renosterveld (Skowno et al., 2019, 2021). Renosterveld is restricted to relativity nutrientrich soils in the south and west of the Western Cape Province. It is noteworthy for its exceptionally high diversity of bulbs and - due to widespread conversion of intact Renosterveld to agriculture - perhaps the highest concentration of plants threatened with extinction in the world (Brummitt et al., 2015; Humphreys et al., 2019; Raimondo, 2011; Skowno et al., 2021). Only 5-10\% its original extent remains, with much of this contained in small isolated fragments (Von Hase et al., 2003). Despite legislation prohibiting the destruction 
of Renosterveld habitat, loss is ongoing (Moncrieff, 2021). It 145 is estimated that at current rates Renosterveld will become ${ }_{146}$ extinct as an ecosystem within the 21st century (Skowno ${ }_{147}$ et al., 2021). The primary cause of this decline is the ex-148 pansion of intensive agriculture (primarily for the cultivation ${ }_{149}$ of grains and oil-seed such as wheat, barely and canola), and ${ }_{150}$ overgrazing by livestock.

The current approach to conservation in the region is clearly ${ }^{152}$ inadequate. A remote sensing-based monitoring system, such ${ }^{153}$ as is available for many forest ecosystems worldwide, may ${ }^{154}$ help to reduce rates of Renosterveld loss by aiding in the ${ }^{155}$ identification of land owners responsible for historical loss ${ }^{156}$ - thereby deterring future habitat destruction. The identifica- ${ }^{157}$ tion of habitat loss through the direct comparison of images ${ }^{158}$ taken taken months or years apart can produce very accurate ${ }^{159}$ maps of change within a discrete time period. A range of ${ }^{160}$ methods are available to achieve this ranging from the very ${ }^{16}$ simple (e.g. direct difference of vegetation indexes) to su- ${ }^{162}$ pervised methods that rely on validated ground truth data ${ }^{163}$ combined with machine learning approaches (Kennedy et al., ${ }^{164}$ 2009). Moncrieff (2021) used a training dataset of validated ${ }^{165}$ land cover change events to produce a map of Renosterveld ${ }^{166}$ loss between 2016 and 2020 after manually verifying poten- ${ }^{167}$ tial change identified through model predictions.

While the direct comparison of paired images provides use- ${ }_{170}$ ful information for monitoring and reporting on habitat loss, ${ }_{171}$ this information is usually only obtained months or years af- ${ }_{172}$ ter the reported change has occurred (Kennedy et al., 2010). ${ }_{173}$ If information is provided with low latency between the on- ${ }_{174}$ set of ploughing and its detection, there may be potential ${ }_{175}$ to intervene while habitat destruction is underway. Contin- ${ }_{176}$ uous change detection approaches attempt to assimilate and ${ }_{177}$ analyze new remote sensing data as they become available, ${ }_{178}$ and have the potential to provide near-real time alerts on land ${ }_{179}$ cover change. A typical pattern used for continuous change ${ }_{180}$ detection involves building a model that can accurately pre- ${ }_{181}$ dict the vegetation activity of intact forests, and compar- ${ }_{182}$ ing predictions to recently acquired observations (Verbesselt ${ }_{183}$ et al., 2012; Ye et al., 2021a; Zhu and Woodcock, 2014; Zhu ${ }_{184}$ et al., 2020). When observed vegetation activity falls outside ${ }_{185}$ out the range expected for natural vegetation, this is taken to be indicative of forest loss. A variety of methods have been used to model expected vegetation activity, ranging from neu- ${ }^{180}$ ral networks to relativity simple linear models (Tang et al., ${ }^{187}$ 2019). The thresholds used to determine whether anomalies 188 are indeed true change are often predetermined heuristically. 189 This is necessary as determining the degree of change in veg- 190 etation activity that is indicative of vegetation cover change 191 from data requires not only maps of where change has oc- 192 curred, but also precise dates of the change events. The re- 193 sulting land cover change detections, while impressive for ${ }_{194}$ stable ecosystems such as forest, are prone to commission 195 errors in dynamic ecosystems where natural variability can 196 easily be mistaken for land cover change and omission errors 197 when disturbance is subtle. This problem is particularly acute ${ }_{198}$ in non-forest ecosystems with natural dynamics such as fire, 199 aridity and herbivory (Slingsby et al., 2020).
If ground-truth data are available, time-series of vegetation activity can be classified into land cover types or land cover change using supervised learning. This approach is still limited to producing updates on land cover change at fixed intervals (typically annually), as the training data typically do not specify the exact date on which change occurred, but rather that change occurred between two fixed points in time (e.g. sometime between January and December). Thus a new full year of data are required before a new prediction can be made. If a precise date for a land cover change event is available, time series classifiers that are robust to seasonal variation and natural variability can be trained (Figure 1). These models can then be applied continuously as new data become available and produce near-realtime land cover change alerts. They would also be less prone to errors than unsupervised change detection methods, as the models could theoretically learn to distinguish the spectral patterns indicative of land cover change from natural disturbances. Hansen et al. (2016) trained decision-tree classifiers to detect forest disturbance in the tropics, comparing recent imagery to a stable reference period. Their system is widely used for forest monitoring in the tropics and has been incredibly impactful. Approaches using neural network architectures such as convolutional neural networks (CNN) or self-attention have shown improved performance on land cover classification tasks relative to more traditional tree-based machine learning approaches, but are not yet widely applied to continuous change detection (Kussul et al., 2017; Pelletier et al., 2019; Rußwurm and Körner, 2019).

Here I describe the design and performance of continuous land cover change detection in a critically endangered shrubland ecosystem, the Renosterveld of South Africa, based on supervised machine learning approaches. Using an dataset of precisely dated land cover change events, a variety of deep learning architectures are trained and evaluated against treebased and unsupervised methods commonly used for land cover change detection and monitoring in forested ecosystems. Models building and design choices are motivated by an intention to produce a system that can be deployed for operationally use by conservation management and law enforcement agencies.

\section{Methods}

The study region is limited to the extent of the Renosterveld bioregion in the Overberg district municipality in the Western Cape Province of South Africa (Mucina and Rutherford, 2006). The choice of region was determined by the critically endangered status of Renosterveld vegetation, the availability of accurate land cover data for the Overberg municipality, and partner conservation organizations in the region. The Overberg municipality covers an area of of $12,241 \mathrm{~km}^{2}$, with Renosterveld covering $4711 \mathrm{~km}^{2}$ with this region naturally. Detailed mapping of remaining natural vegetation in the Overberg based on 2003 data identified $656 \mathrm{~km}^{2}$ of the original extent of Renosterveld vegetation in the Overberg remaining.

Using supervised change detection on Sentinel 2 imagery and 

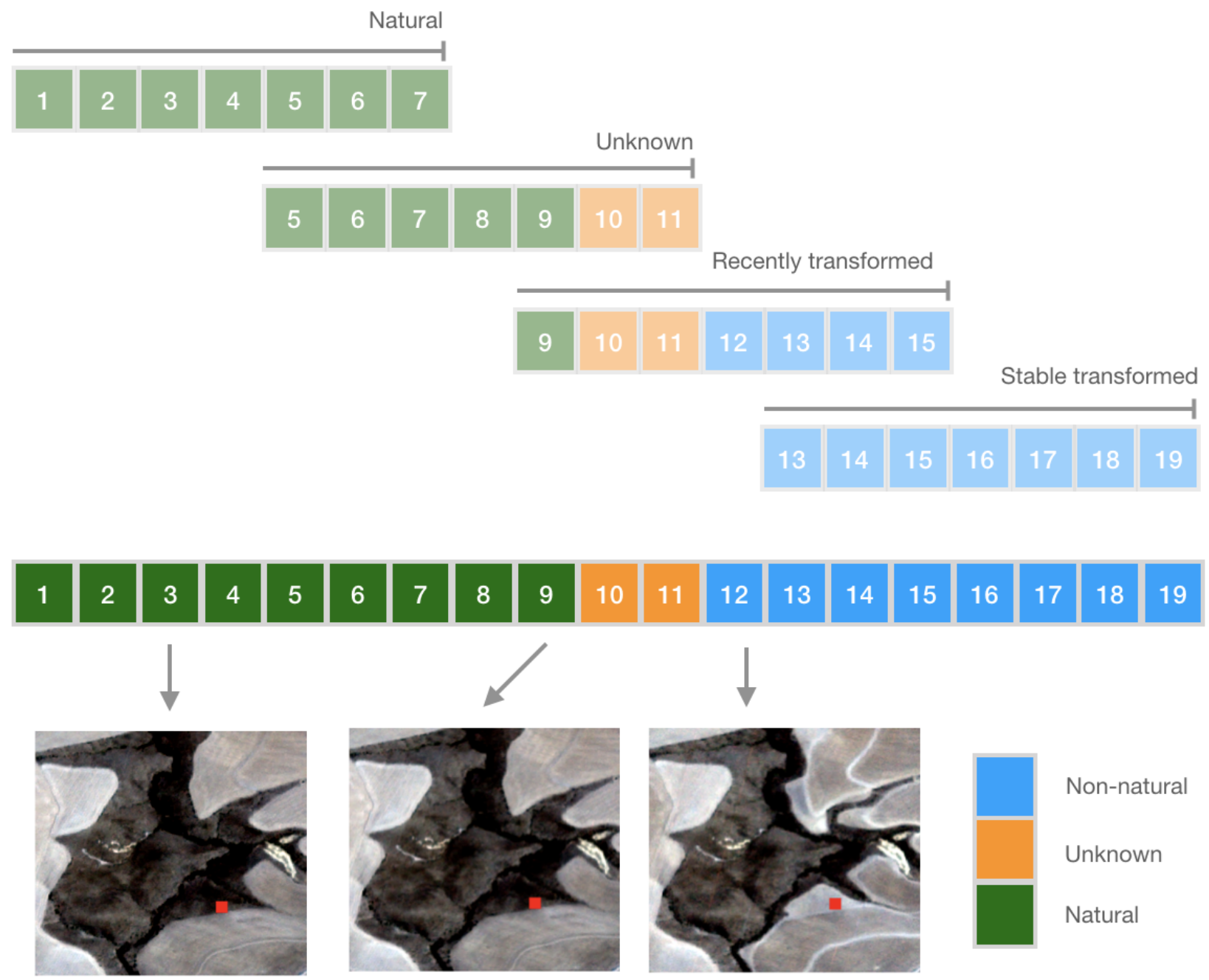

Fig. 1. The approach used for assigning labels to time series. Each step in the sequence of 19 observations represents an observation obtained for a single pixel at a regular interval (e.g. 10 days). The land cover label for the pixel (identified by the red point in the images) can either be natural, unknown or non-natural. This label is obtained through inspection of time series' of Planet Labs PlanetScope satellite imagery. For the example pixel indicated here, an image obtained at time step 3 indicates that the land cover is natural on this date, the image at time step 9 indicates the last date on which natural land cover is observed. The first time at which non-natural land cover is observed is step 12. Hence, it is not known whether the land cover was natural or non-natural at timestep 10 and 11 . Using a window length of 7 observations and forward step of 4,4 labelled sequences can be extracted from the full time series. Each sequence is assigned the label of the most recent observation.

subsequent detailed verification with very high resolution im- 218 agery, Moncrieff (2021) identified all conversion of Renos- 219 terveld to non-natural land cover between January 2016 and 220 January 2020. Land cover change was identified within 268221 spatially contiguous parcels totalling 478.6 ha. Using daily 222 Planet Labs PlanetScope data, dates were assigned to each 223 event indicating the closest possible timing for the occurrence 224 of the land cover change event. It is not possible to assign 225 every event to a single day as often multiple days or even 226 weeks elapse between the beginning and end of a land cover 227 change event. Furthermore, clouds and haze can reduce the 228 frequency at which clear observations are available. Thus for ${ }_{229}$ each land cover change event two dates were assigned - the 230 latest date on which the presence of natural vegetation could ${ }_{231}$ be confirmed, and the earliest date on which the absence of 232 natural vegetation could be confirmed. These two dates occur within one week for 21 percent of all events, and within two ${ }_{234}^{233}$ months for 87 percent. Here a sample of these data are used where land cover changes dates were assigned with the highest confidence, leaving 185 events and 219 ha of land cover change. An additional 4 events covering 7 ha were identified within the same region between Jan 2020 and Jan 2021. These events were used for testing model performance outside of the temporal range of training data, providing a more accurate assessment of operational performance. To augment the no change data parcels of vegetation that remained natural over the entire study period were added. This added an additional 2590 ha to the training and validation dataset and 24 ha to the testing dataset. These no-change parcels were selected from the (Von Hase et al., 2003) map of remaining Renosterveld, buffered to ensure that they did not occur within $100 \mathrm{~m}$ of non-natural vegetation and manually verified.

Input time series' for classification were created using Sentinel 2 L1C data (S2). For training and validation all available 
S2 data up until 2019-12-31 were extracted and preprocessed. 292 S2 images are pre-screened and discarded if total scene cloud 293 cover exceeds 50 percent. Pixel-level cloud masking is per- 294 formed using the s2cloudless machine learning-based cloud 295 masks. Pixels with a cloud probability greater than 40 per- 296 cent are masked. Cloud shadows are also masked and are 297 calculated from cloud masks using cloud and solar geome- ${ }_{298}$ try combined with dark pixel detection. All S2 bands are ${ }_{299}$ retained and the following additional vegetation indices cal- ${ }_{300}$ culated: NDVI, EVI, NDWI, NBR, NDRE. Data are exported ${ }_{301}$ at $10 \mathrm{~m}$ resolution with $20 \mathrm{~m}$ and $60 \mathrm{~m}$ bands resampled using bilinear interpolation.

S2 data area available every 10 days in this region until mid- 304 2017 when data become available at least every 5 days sub- 305 sequent to the launch of Sentinel 2B. In parts of the study 306 region data is available at frequencies higher than 5 days due 307 to swath overlap. To create regular time series from the ir- 308 regularly spaced S2 image stacks pixel-level time series are 309 created with a single observation every 10 days. Where mul- 310 tiple images are available within a 10 day period, data from 311 the image with the highest pixel-level NDVI value are used. 312 Where no data is available, the reflectance and vegetation in- 313 dices from the previous valid image is used unaltered. $\mathrm{In}_{314}$ this application interpolation between observations by aver- ${ }_{315}$ aging is undesirable as it may obfuscate sudden changes in ${ }_{316}$ reflectance diagnostic of land cover change. Classification ${ }_{317}$ is performed on time series of reflectance collected over $180_{318}$ days, equalling 18 observations with a 10 day interval. The ${ }_{319}$ choice of 180 days was arrived upon through experimentation ${ }_{320}$ with both longer and short time series'.

The time series for each pixel is assigned the label 'natural' ${ }_{222}$ if the last date of confirmed natural vegetation falls after the ${ }^{323}$ end date of the time series under consideration (Figure 1). If 324 both the last date of confirmed natural vegetation and the first 325 date of confirmed land cover change occur within the 180 day ${ }^{2} 6$ time series' the land cove change event must have occurred ${ }^{327}$ within the time series under consideration and the 'recently ${ }_{2} 8$ transformed' label is assigned. If the last date of confirmed ${ }^{329}$ natural vegetation occurs within the time series and the first 330 date of confirmed land cover change falls after the end of ${ }_{331}$ the time series' it is unclear whether land cover change has ${ }_{332}$ indeed taken place and the data point is discarded. Similarly, if the first date of confirmed land cover change occur before ${ }_{334}$ the start of the time series, land cover change has already ${ }_{335}$ taken place. Here there is no need to identify stable non- ${ }_{336}$ natural land cover, thus these data are discarded.

The resulting labelled dataset is highly imbalanced, with the ${ }^{338}$ vast majority of samples in the 'natural' class. There is also ${ }^{339}$ very high spatial auto-correlation among neighbouring pix- 340 els within the same parcel. Successive time series for the ${ }^{341}$ same pixel obtained 10 days apart will share 94 percent of ${ }^{342}$ observations. To ameliorate class imbalance data and spatial ${ }^{343}$ autocorrelation only 5 percent of pixels within parcels where ${ }^{344}$ no change occurs are sampled and 33 percent of pixels from ${ }^{345}$ parcels where change does occur are used. Subsequent to this ${ }_{346}$ data thinning, parcels are assigned to training and validation ${ }_{347}$ sets using a 70/30 split. To reduce temporal oversampling ${ }_{348}$ within a pixel, time series windows of 180 days are shifted forward by 60 days for every sample taken. This represents a trade off between reducing temporal oversampling and discarding labelled data. Test data does not undergo temporal or spatial thinning, and a new prediction is made every 10 days to quantify performance in an operational setting.

Two neural network architectures that have shown excellent performance in classification of land cover from satellite image times series are trained and evaluated along with random forests, a tree based method commonly used for land cover classification. TempCNN is a $1 \mathrm{D}$ convolutional neural network for land cover classification from satellite image times series (Pelletier et al., 2019). Convolutional kernels that extract relevant features in local temporal regions across multivariate time series of individual pixels are learned. These 1D CNN layers are followed by non-linear activations and potentially also batch normalization and stacked, before class probabilities are produced by a dense and softmax layer. TempCNN performs well on a range of land cover classifications tasks, and has been adopted by multiple packages for classification of satellite image time series (Rußwurm et al., 2020a; Simoes et al., 2021).

Transformer models were originally proposed as sequenceto-sequence encoder-decoder models based on the selfattention operation and applied to language translation (Vaswani et al., 2017). Attention mechanisms operate by assigning an importance-score (attention) to weight each element in a sequence (Bahdanau et al., 2016). In self-attention, the concept of attention is used to encode sequences to a hidden representation. In the traditional application of transformers to language tasks, self-attention encoders are followed by self-attention decoders. For time series classification only the encoder network is needed. In this work, a Transformer model that has been evaluated in (Rußwurm and Körner, 2019) is used, where individual pixels' time series' are encoded using stacked self-attention layers. This architecture outperforms many other neural networks and treebased methods for crop type mapping on satellite image time series (Rußwurm et al., 2020a).

The accurately dated land cover change data used here to train supervised methods is seldom available. Unsupervised approaches to land cover change detection are more generally applicable and widely used (Bullock et al., 2020; Tang et al., 2019). A simple unsupervised baseline is used to evaluate the improvement in accuracy achieved when using supervised methods on these data. The approach followed to construct the unsupervised baseline is very similar to the approached outlined by Zhu and Woodcock (2014), termed Continuous Change Detection and Classification (CCDC). CCDC is widely used for continuous change detection, and implemented in platforms such as Google Earth Engine. More recent unsupervised methods that would improve on this baseline are available (Ye et al., 2021a,b; Zhou et al., 2019), but they are not commonly used on S2 data.

The unsupervised baseline operates by fitting a simple linear model with a single harmonic term to capture seasonal variation using robust regression through iteratively reweighted 
Table 1. Model performance evaluated on test data. Recall-1-short reports model recall when change has occured within the first 2 time-steps (20 days), of the 18 step (180 days) time series. Performance of the top-performing model for each metric is highlighted in bold.

\begin{tabular}{llllllll}
\hline & Accuracy & F1 & Recall-1 & Recall-0 & Precision-1 & Precision-0 & Recall-1-short \\
\hline Random Forest & 0.98 & 0.88 & 0.79 & $\mathbf{0 . 9 9}$ & $\mathbf{0 . 9 8}$ & 0.98 & \\
TempCNN & $\mathbf{0 . 9 9}$ & $\mathbf{0 . 9 3}$ & $\mathbf{0 . 8 9}$ & $\mathbf{0 . 9 9}$ & 0.96 & $\mathbf{0 . 9 9}$ & $\mathbf{0 . 6 4}$ \\
Transformer & $\mathbf{0 . 9 9}$ & 0.92 & 0.88 & $\mathbf{0 . 9 9}$ & 0.97 & $\mathbf{0 . 9 9}$ & 0.60 \\
CCDC & 0.93 & 0.57 & 0.48 & 0.98 & 0.70 & 0.94 & - \\
\hline
\end{tabular}

least squares with the Talwar cost function. The model is fit- 396 ted to historical NDVI data for every pixel in regions known ${ }_{397}$ to still contain Renosterveld. The expected NDVI over the 398 test period (2020-01-01 - 2020-12-31) is then calculated for 399 each day of the year at each pixel. This is compared to the 400 observed NDVI and anomalies calculated and expressed in 401 terms of the RMSE of the fitted regression. If 3 successive 402 observations exceed the prediction by 3 times the RMSE, 403 land cover change is assumed to have occurred. More sophis- 404 ticated implementations of this workflow make use of multi- ${ }_{405}$ ple bands, or use parameters learned from labelled land cover ${ }_{406}$ change events (Ye et al., 2021b).

Hyperparameter optimization for random forests, TempCNN ${ }_{408}$ and the Transformer network is performed using a grid search ${ }_{409}$ over 24,36 and 32 parameter combinations respectively. The ${ }_{410}$ best fitting model of each class is then evaluated against ${ }_{411}$ test data. For the Transformer and TempCNN, the Adam ${ }_{412}$ optimizer is used is minimize focal loss. TempCNN and Transfomers as implemented in Tensorflow 2 and the eo-flow ${ }^{413}$ python package. scikit-learn is used for Random Forests. ${ }^{414}$ Google Earth Engine is used for preprocessing of input data ${ }_{416}^{415}$ (Gorelick et al., 2017).

To aid model interpretation, the most important temporal re- ${ }^{417}$ gions identified by the TempCNN model for detecting land ${ }^{41}$ cover change are calculated using Grad-CAM++ (Chattopad- ${ }^{419}$ hay et al., 2018). Originally developed for use with $\mathrm{CNNs}^{420}$ and image classification, Grad-CAM++ can easily be used ${ }^{421}$ when CNNs are applied to time series classification. The im- ${ }^{422}$ portance of each time-step in the classification of a time series ${ }^{423}$ is calculated using a weighted average of all positive activa- ${ }^{424}$ tion gradients across channels in the final convolutional layer. ${ }^{425}$

\section{Results}

The best performing TempCNN model used a dropout rate ${ }_{429}$ of $0.8, \mathrm{~L} 2$ kernel regularization with a regularization factor 430 of 1e-8, He normal kernel initialization, 6 stacked convolu- 431 tional layers each with 32 filter and 256 neurons in the fi- 432 nal fully connected layer. The best performing Transformer ${ }_{433}$ used a dropout rate of $0.5,4$ layers in the encoder with $4{ }_{434}$ attention heads, a model depth/query size of 128 , no layer 435 normalization and 256 neurons in the encoder feed-forward ${ }_{436}$ layer. The best performing random forest model used $100_{437}$ estimators, and required a minimum of 4 samples to split an ${ }_{438}$ internal node.

The best performing supervised model was the TempCNN, ${ }_{440}$ with a recall of 0.89 and precision of 0.96 when identifying ${ }_{441}$ Renosterveld loss and overall f-score of 0.93 (Table 1). The 442 Transformer was only slightly worse than the TempCNN with ${ }_{443}$ a recall of 0.88 and precision of 0.97 on the Renosterveld loss class and overall f-score of 0.92, while the Random Forest was significantly worse than both TempCNN and the Transfomer, with Renosterveld loss recall and precision of 0.79 and 0.98 , and overall f-score of 0.88 . All supervised models outperformed unsupervised classification, which was far more likely to produce both false negatives and false positives with recall of 0.48 and precision of 0.70 on the Renosterveld loss class and overall f-score of 0.57 .

Performance for all supervised models declines when their ability to detect Renosterveld loss within 20 days of change is measured. Again the TempCNN performed best, with recall of 0.64 , followed by the Transformer with 0.60 and the random forest with 0.50 . Unsupervised classification is not possible within 20 days of change, as 3 anomalous data points are required before change is flagged. This equates to 30 days with the 10 day interval used here.

Figure 2 provides a visual depiction of the time series features characteristic of Renosterveld loss events, determined using Grad-CAM++ on the trained TempCNN. For a single Renosterveld loss event in a pixel viewed from multiple points in time after the event, the same temporal features are focused upon. Across multiple different land cover change events, the network has learnt to consistently focus on time periods in which a drop in vegetation greenness has occurred. Other more nuanced changes in spectral reflectance may also be contributing to the saliency of these temporal regions.

A prototype application performing up-to-date inference over the entire study region every 10 days using TempCNN is available at https://glennwithtwons.users.earthengine.app/view/globalrenosterveld-watch and an example sequence of predictions over 2 months in an area throughout course of a land cover change event is depicted in Figure 3. Inference over the entire remaining distribution of Renosterveld in the Overberg region for a single date reveals multiple as-yet unreported areas where Renosterveld has been transformed to cropland within the 6 month time series of satellite data under consideration. Because models are trained to only make predictions in regions where land cover is intact Renosterveld at the beginning of the time series under consideration, predictions are not reliable when made on other vegetation types, or where Renosterveld has already been converted to agriculture. This is not an issue when up-to-date land cover maps are available. Multiple observed cases of incorrect predictions of Renosterveld loss where land cover has in reality remained as agriculture throughout the time series under consideration highlight the need for 
a

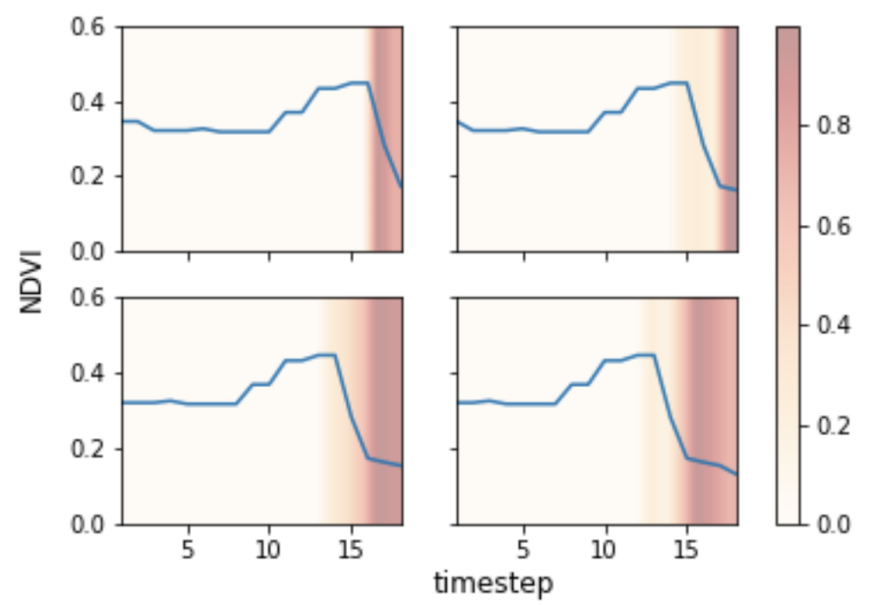

b

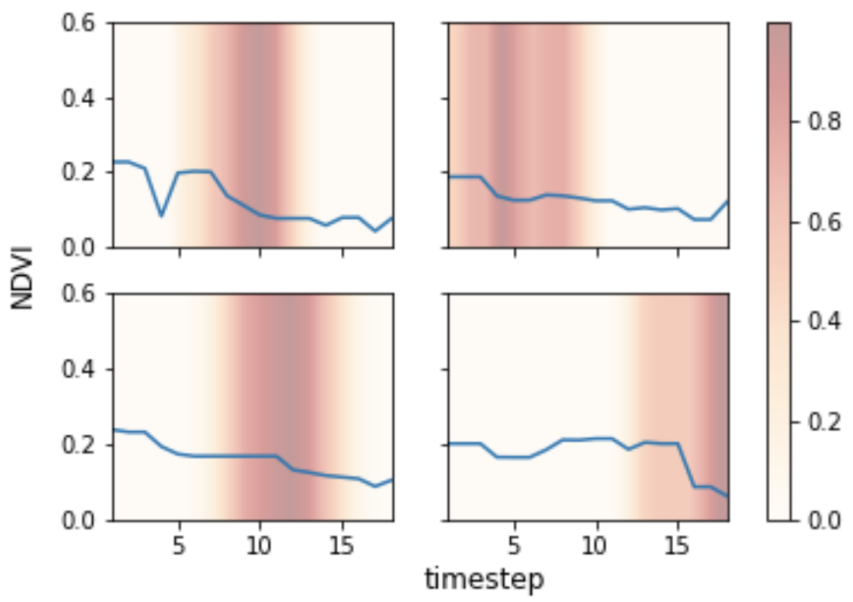

Fig. 2. Saliency of temporal regions in time series for pixels in which land cover change is predicted to have occurred, determined using Grad-CAM++. Higher saliency scores indicates that the region is more influential in determining that land cover change has occurred. Blue lines show NDVI, though saliency is calculated using all input features. In a) a single pixel is shown at four points in time, with each subsequent panel shifted forward 1 step (10 days). In b) time series' from four spatially and temporal independent pixels are shown.

updating of existing regional land cover maps.

\section{Discussion}

Shrublands are globally important repositories of biodiversity and play an important role in regulating planetary biochemical cycles (Bond, 2019; Poulter et al., 2014). In many regions shrublands are being lost at a rate that exceeds other ecosystems such as forests (Parr et al., 2014). Despite this, few studies have attempted to develop algorithms designed specifically for operational monitoring of shrubland loss, ostensibly because many of these ecosystem display complex natural dynamics and have low overall productivity. These characteristics make the unsupervised methods typically applied in operational forest monitoring perform poorly. This study ${ }^{491}$ demonstrates that reliable detection of land cover change in ${ }^{492}$ shrublands in possible, and that detection is possible within ${ }^{493}$ days of land cover change events, thus facilitating the use of ${ }^{494}$ these algorithms for operational monitoring and enforcement. ${ }^{495}$ The key component of this is the use of supervised classifi- ${ }^{496}$ cation, enabled by the availability of hand-labelled change ${ }^{497}$ events with the date of each event precisely determined. $\quad{ }_{498}$ Supervised models for continuous detection of land cover ${ }^{499}$ change are rare. The labels needed to train them require de- ${ }^{500}$ tailed examination of very-high resolution satellite or aerial ${ }^{501}$ imagery and dense time series. Very-high resolution data ${ }^{502}$ may not be available for the required region, time period or ${ }^{503}$ frequency, and the costs associated with these will be pro- ${ }^{504}$ hibitive for many potential use cases. Even when all the nec- ${ }^{505}$ essary data is available, the data labeller must have intimate ${ }^{506}$ knowledge of the ecosystem in question in order to deter- ${ }^{507}$ mine whether an observed event is indeed land cover change. ${ }^{508}$ For example, Renosterveld is a naturally fire-prone ecosys- ${ }^{509}$ tem and burnt vegetation is not necessarily indicative of land ${ }_{510}$ cover change. These limitations should not, however, pre- 511 clude the development of labelled datasets. The availability 512$$
\text { va }
$$

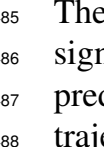

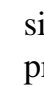
change monitoring system will slow or reverse these trends.

The volume of labels required to achieve a performant model in the example presented here is surprisingly small - just 219 ha of dated land cover change events. This is sufficient to produce large improvements in performance in deep learningbased classifiers relative to random forests which typically require less data to train. While it is highly unlikely that a supervised approach will be applicable in any region beyond the domain of the training data - thus requirement further painstaking data collection and labelling - meta-learning approaches ought to reduce data requirements for application to new disturbance types and new geographies (Vanschoren, 2019). Given multiple labelled datasets of dated land cover change from different geographies or ecosystems, models can be trained such that they are initialized optimally and learn rapidly when confronted with a new regime. Particularly promising is model-agnostic meta-learning, which does not necessitate custom designed models for this task, but rather can make use of widely adopted models such as TempCNN or Transformers (Finn et al., 2017; Rußwurm et al., 2020b).

A major improvement achieved through the approach presented here is the ability to detect change within a few days of the actual event. Rapid identification is critical in 
bioRxiv preprint doi: https://doi.org/10.1101/2021.10.26.465837; this version posted October 28, 2021. The copyright holder for this preprint (which was not certified by peer review) is the author/funder, who has granted bioRxiv a license to display the preprint in perpetuity. It is made available under aCC-BY-ND 4.0 International license.
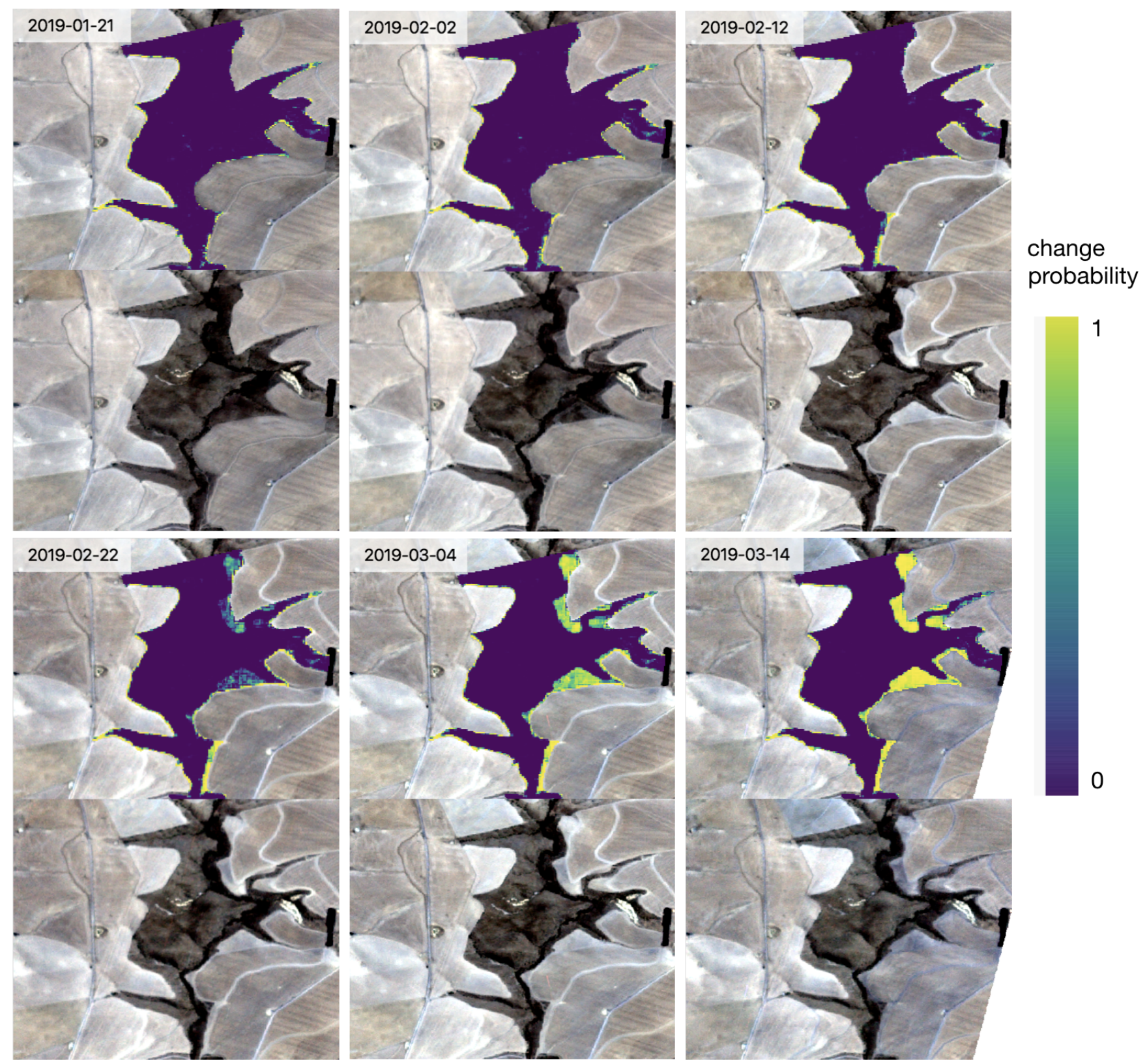

Fig. 3. Example sequence of predictions through the course of a land cover change event in Renosterveld shrublands of the Overberg region, South Africa. Upper panels show the predicted probability of land cover change, where predictions are only made in pixels known to have intact natural vegetation at the start of the monitoring period. The lower panels show the most recent Planet labs PlanetScope imagery collected prior to, or on the date for which predictions are made. The detection of Renosterveld loss along the eastern edge of the monitored area is apparent. This detection occurs within 10-20 days of the actual land cover change event.

many intended applications of near-realtime continuous land ${ }_{526}$ cover change monitoring - such as law enforcement and ${ }_{527}$ management intervention. Unsupervised approaches typically require multiple consecutive observations confirming ${ }_{528}$ the change has occurred before a positive identification is 529 made (Ye et al., 2021a; Zhu and Woodcock, 2014). Gen- 530 erally any land cover change will be complete by the time ${ }_{531}$ these detections are reported. Here change is identified with 532 relative skill with only 2 observation subsequent to the actual 533 change event. In certain applications this may be sufficient to 534 lead to an on-the-ground intervention that could preventing ${ }_{535}$ further damage. Accuracy improves as further observations 536 are acquired, and hence it is possible to delay the identifica- 537 tion of change depending on the cost/benefit of any particular application.

The improvement in performance obtained when using deep learning-based time-series classifiers vs. Random forests is likely a result of their ability to retain temporal information and extract features based thereon. Previous work comparing the performance of Transformers and TempCNN for land cover classification found Transformers to generally perform better than TempCNN, particularly when minimal prepossessing and no atmospheric correction is performed (Rußwurm and Körner, 2019; Rußwurm et al., 2020a). Here, extensive feature engineering has been conducted and cloud masking performed to remove noisy data, potentially result- 
bioRxiv preprint doi: https://doi.org/10.1101/2021.10.26.465837; this version posted October 28, 2021. The copyright holder for this preprint (which was not certified by peer review) is the author/funder, who has granted bioRxiv a license to display the preprint in perpetuity. It is made available under aCC-BY-ND 4.0 International license.

ing in the convergence in performance between TempCNN 600 and the Transformer. A range of machine learning methods ${ }_{602}^{601}$ that are not based on neural networks have been developed ${ }_{003}^{602}$ for the classification of time-series data, and there may be ${ }^{604}$ potential performance gains when using these methods, par- 606 ticularly when training data is scarce (Dempster et al., 2020; ${ }_{608}^{607}$ Maus et al., 2016). Gains in performance cannot, however, 609 come at the costs of increases in model inference time to ${ }_{611}^{610}$ the extent that prediction cannot be made at the required la- 612 tency or within available budgets. This is particularly rel- ${ }_{614}^{613}$ evant because monitoring will often be required over very 615 large regions. Using the TempCNN model fitted here pre- ${ }_{617}^{616}$ diction over the entire remaining range of Renosterveld in ${ }_{618}^{617}$ the Overberg region can be achieved within 120 CPU hours. ${ }^{619}$ The full prediction pipeline for preprocessing, prediction and 621 postprocessing is achieved using tools such as Google Earth ${ }^{622}$ Engine and Google Cloud Dataflow.

\section{Conclusions}

The potential of supervised continuous change detection us- ${ }^{629}$ ing deep learning on satellite image time series, particularly ${ }_{631}$ in non-forest ecosystems, remains largely unexploited. This ${ }_{633}^{632}$ study demonstrates that when labelled data are available, ac- ${ }^{634}$ curate and precise detection of land cover change is possible ${ }_{636}^{635}$ at low latency. Obtaining the labelled data does indeed re- ${ }^{637}$ quire significant effort, and is likely that independent datasets ${ }_{639}^{638}$ and models for will be required for different ecosystems. 640 This should not discourage the further development and op- ${ }_{642}^{641}$ erationalization of this approach. Both deep learning and the ${ }^{443}$ explosion of remote sensing data have been lauded as having ${ }_{645}^{644}$ great potential to address the biodiversity and climate crises. ${ }^{466}$ For these technologies to truly have impact they must find ${ }_{648}^{647}$ application beyond the forested ecosystems of the world, and 649 embrace the complexity of open ecosystems.

\section{ACKNOWLEDGEMENTS}

I am very grateful for the support and encouragement given by Odette Curtis and 654 her passion for conservation in this region. Marcel Gietzmann-Sanders helped pro- 655 duce the prototype operational monitoring application. Multiple individuals from 656 the Western Cape Department of Environmental Affairs and Development Planning 657 are acknowledged for their input, in particular, those from the Environmental Law 658 Enforcement directorate. This research was supported by the National Research 659 Foundation of South Africa through (Grant No. 118593) as part of the RReTool: 660 Rapid and repeatable tools for monitoring and mitigating global change impacts 661 on natural resources project, and the Group on Earth Observations-Google Earth 662 Engine Programme. The funders had no role in study design, data collection and 663 analysis, decision to publish, or preparation of the manuscript.

\section{DATA AVAILABILITY}

Code and data used in this analysis are available at ${ }_{667}^{667}$ https://github.com/GMoncrieff/renosterveld-monitor. Due to ongoing criminal 660 investigations location data has been removed to protect the identity of landowners ${ }_{670}^{69}$ where necessary.

\section{Bibliography}

D. Bahdanau, K. Cho, and Y. Bengio. Neural Machine Translation by Jointly Learning to Align and 676 Translate. arXiv:1409.0473 [cs, stat], May 2016. URL http://arxiv.org/abs/1409. 677 0473. arXiv: 1409.0473 .

W. J. Bond. Open ecosystems: ecology and evolution beyond the forest edge. Oxford University 679 Press, 2019.

E. S. Brondizio, J. Settele, S. Díaz, and H. T. Ngo. Global assessment report on biodiversity 681 and ecosystem services of the Intergovernmental Science-Policy Platform on Biodiversity and 682
Ecosystem Services. 2019.

N. A. Brummitt, S. P. Bachman, J. Griffiths-Lee, M. Lutz, J. F. Moat, A. Farjon, J. S. Donald- 684 son, C. Hilton-Taylor, T. R. Meagher, S. Albuquerque, E. Aletrari, A. K. Andrews, G. Atchison, 685
E. Baloch, B. Barlozzini, A. Brunazzi, J. Carretero, M. Celesti, H. Chadburn, E. Cianfoni, C. Cockel, V. Coldwell, B. Concetti, S. Contu, V. Crook, P. Dyson, L. Gardiner, N. Ghanim, H. Greene, A. Groom, R. Harker, D. Hopkins, S. Khela, P. Lakeman-Fraser, H. Lindon, H. Lockwood, C. Loftus, D. Lombrici, L. Lopez-Poveda, J. Lyon, P. Malcolm-Tompkins, K. McGregor, L. Moreno, L. Murray, K. Nazar, E. Power, M. Q. Tuijtelaars, R. Salter, R. Segrott, H. Thacker, L. J. Thomas, S. Tingvoll, G. Watkinson, K. Wojtaszekova, and E. M. N. Lughadha. Green Plants in the Red: A Baseline Global Assessment for the IUCN Sampled Red List Index for Plants. PLOS ONE, 10(8):e0135152, Aug. 2015. ISSN 1932-6203. doi: 10.1371/journal.pone.0135152. URL https://journals.plos.org/plosone/ article?id=10.1371/ journal. pone.0135152.

E. L. Bullock, C. E. Woodcock, and C. E. Holden. Improved change monitoring using an ensemble of time series algorithms. Remote Sensing of Environment, 238:111165, Mar. 2020. ISSN 0034-4257. doi: 10.1016/j.rse.2019.04.018. URL https://www.sciencedirect.com/ science/article/pii/s0034425719301634.

A. Chattopadhay, A. Sarkar, P. Howlader, and V. N. Balasubramanian. Grad-cam++: Generalized gradient-based visual explanations for deep convolutional networks. In 2018 IEEE winter conference on applications of computer vision (WACV), pages 839-847. IEEE, 2018.

A. Dempster, F. Petitjean, and G. I. Webb. ROCKET: exceptionally fast and accurate time series classification using random convolutional kernels. Data Mining and Knowledge Discovery, 34 (5):1454-1495, 2020.

C. Finn, P. Abbeel, and S. Levine. Model-agnostic meta-learning for fast adaptation of deep networks. In International Conference on Machine Learning, pages 1126-1135. PMLR, 2017.

N. Gorelick, M. Hancher, M. Dixon, S. Ilyushchenko, D. Thau, and R. Moore. Google Earth Engine: Planetary-scale geospatial analysis for everyone. Remote sensing of Environment, 202:18-27, 2017.

M. C. Hansen, A. Krylov, A. Tyukavina, P. V. Potapov, S. Turubanova, B. Zutta, S. Ifo, B. Margono, F. Stolle, and R. Moore. Humid tropical forest disturbance alerts using Landsat data. 11(3): 034008, Mar. 2016. ISSN 1748-9326. doi: 10.1088/1748-9326/11/3/034008. URL https: //doi.org/10.1088/1748-9326/11/3/034008.

A. M. Humphreys, R. Govaerts, S. Z. Ficinski, E. N. Lughadha, and M. S. Vorontsova. Global dataset shows geography and life form predict modern plant extinction and rediscovery. Nature Ecology \& Evolution, 3(7):1043-1047, July 2019. ISSN 2397334X. doi: 10.1038/s41559-019-0906-2. URL https://www. nature.com/articles/ s41559-019-0906-2.

R. E. Kennedy, P. A. Townsend, J. E. Gross, W. B. Cohen, P. Bolstad, Y. Q. Wang, and P. Adams. Remote sensing change detection tools for natural resource managers: Understanding concepts and tradeoffs in the design of landscape monitoring projects. Remote sensing of environment, 113(7):1382-1396, 2009.

R. E. Kennedy, Z. Yang, and W. B. Cohen. Detecting trends in forest disturbance and recovery using yearly Landsat time series: 1. LandTrendr - Temporal segmentation algorithms. Remote Sensing of Environment, 114(12):2897-2910, Dec. 2010. ISSN 0034-4257. doi: 10.1016/j.rse.2010.07.008. URL http://www.sciencedirect.com/science/ article/pii/s0034425710002245.

N. Kussul, M. Lavreniuk, S. Skakun, and A. Shelestov. Deep learning classification of land cover and crop types using remote sensing data. IEEE Geoscience and Remote Sensing Letters, 14(5):778-782, 2017.

J. J. Le Roux, C. Hui, M. L. Castillo, J. M. Iriondo, J.-H. Keet, A. A. Khapugin, F. Médail, M. Rejmánek, G. Theron, F. A. Yannelli, and H. Hirsch. Recent Anthropogenic Plant Extinctions Differ in Biodiversity Hotspots and Coldspots. Current Biology, 29(17):29122918.e2, Sept. 2019. ISSN 0960-9822. doi: 10.1016/j.cub.2019.07.063. URL https: //www. sciencedirect.com/science/article/pii/s0960982219309431.

J. Manning and P. Goldblatt. Plants of the Greater Cape Floristic Region. 1: The Core Cape flora. South African National Biodiversity Institute, 2012.

V. Maus, G. Câmara, R. Cartaxo, A. Sanchez, F. M. Ramos, and G. R. De Queiroz. A timeweighted dynamic time warping method for land-use and land-cover mapping. IEEE Journal of Selected Topics in Applied Earth Observations and Remote Sensing, 9(8):3729-3739, 2016.

G. R. Moncrieff. Locating and Dating Land Cover Change Events in the Renosterveld, a Critically Endangered Shrubland Ecosystem. Remote Sensing, 13(5):834, Jan. 2021. doi: 10.3390/ rs13050834. URL https://www.mdpi.com/2072-4292/13/5/834.

L. Mucina and M. C. Rutherford, editors. The vegetation of South Africa, Lesotho and Swaziland. Strelitzia 19. South African National Biodiversity Institute, Pretoria., 2006.

N. Myers, R. A. Mittermeier, C. G. Mittermeier, G. A. B. da Fonseca, and J. Kent. Biodiversity hotspots for conservation priorities. Nature, 403(6772):853-858, Feb. 2000. ISSN 1476-4687. doi: 10.1038/35002501. URL https://www. nature.com/articles/35002501.

C. L. Parr, C. E. Lehmann, W. J. Bond, W. A. Hoffmann, and A. N. Andersen. Tropical grassy biomes: misunderstood, neglected, and under threat. Trends in ecology \& evolution, 29(4): 205-213, 2014.

C. Pelletier, G. I. Webb, and F. Petitjean. Temporal Convolutional Neural Network for the Classification of Satellite Image Time Series. Remote Sensing, 11(5):523, Jan. 2019. doi: 10.3390/rs11050523. URL https: //www. mdpi.com/2072-4292/11/5/523.

B. Poulter, D. Frank, P. Ciais, R. B. Myneni, N. Andela, J. Bi, G. Broquet, J. G. Canadell, F. Chevallier, Y. Y. Liu, S. W. Running, S. Sitch, and G. R. van der Werf. Contribution of semi-arid ecosystems to interannual variability of the global carbon cycle. Nature, 509 (7502):600-603, May 2014. ISSN 1476-4687. doi: 10.1038/nature13376. URL https : //www.nature.com/articles/nature13376.

D. Raimondo. The Red List of South African plants: a global first. South African Journal of Science, 107(3-4):01-02, Apr. 2011. ISSN 0038-2353. doi: 10.4102/sajs.v107i3/ 4.653. URL http://www.scielo.org.za/scielo.php?script=sci_abstract \& pid $=S 0038-23532011000200003 \& l n g=e n \& n r m=i$ so\& $t$ lng $=e n$.

M. Rußwurm and M. Körner. Self-attention for raw optical satellite time series classification. arXiv preprint arXiv:1910.10536, 2019.

M. Rußwurm, C. Pelletier, M. Zollner, S. Lefèvre, and M. Körner. BreizhCrops: A Time Series Dataset for Crop Type Mapping. arXiv:1905.11893 [cs, stat], May 2020a. URL http:// arxiv.org/abs/1905.11893. arXiv: 1905.11893.

M. Rußwurm, S. Wang, M. Korner, and D. Lobell. Meta-learning for few-shot land cover classifi- 
bioRxiv preprint doi: https://doi.org/10.1101/2021.10.26.465837; this version posted October 28, 2021. The copyright holder for this preprint (which was not certified by peer review) is the author/funder, who has granted bioRxiv a license to display the preprint in perpetuity. It is made available under aCC-BY-ND 4.0 International license.

cation. In Proceedings of the ieee/cvf conference on computer vision and pattern recognition workshops, pages 200-201, 2020b.

R. Simoes, G. Camara, G. Queiroz, F. Souza, P. R. Andrade, L. Santos, A. Carvalho, and K. Ferreira. Satellite Image Time Series Analysis for Big Earth Observation Data. Remote Sensing, 13(13):2428, Jan. 2021. doi: 10.3390/rs13132428. URL https://www.mdpi.com/ 2072-4292/13/13/2428.

A. J. Skowno, C. J. Poole, and D. C. Raimondo. National biodiversity assessment 2018: the status of South Africa's ecosystems and biodiversity. Synthesis Report. South African National Biodiversity Institute, Pretoria, 2019.

A. L. Skowno, D. Jewitt, and J. A. Slingsby. Rates and patterns of habitat loss across South Africa's vegetation biomes. South African Journal of Science, 117(1/2), Jan. 2021. ISSN 1996-7489. doi: 10.17159/sajs.2021/8182. URL https://sajs.co.za/article/view/8182.

J. A. Slingsby, G. R. Moncrieff, and A. M. Wilson. Near-real time forecasting and change detection for an open ecosystem with complex natural dynamics. ISPRS Journal of Photogrammetry and Remote Sensing, 166:15-25, Aug. 2020. ISSN 0924-2716. doi: 10.1016/j. isprsjprs.2020.05.017. URL http://www.sciencedirect.com/science/article/ pii/s092427162030143X.

X. Sun, B. Wang, Z. Wang, H. Li, H. Li, and K. Fu. Research progress on few-shot learning for remote sensing image interpretation. IEEE Journal of Selected Topics in Applied Earth Observations and Remote Sensing, 14:2387-2402, 2021.

X. Tang, E. L. Bullock, P. Olofsson, S. Estel, and C. E. Woodcock. Near real-time monitoring of tropical forest disturbance: New algorithms and assessment framework. Remote Sensing of Environment, 224:202-218, 2019.

W. Turner. Sensing biodiversity. Science, 346(6207):301-302, 2014.

W. Turner, S. Spector, N. Gardiner, M. Fladeland, E. Sterling, and M. Steininger. Remote sensing for biodiversity science and conservation. Trends in ecology \& evolution, 18(6):306-314 2003.

J. Vanschoren. Meta-learning. In Automated Machine Learning, pages 35-61. Springer, Cham, 2019.

A. Vaswani, N. Shazeer, N. Parmar, J. Uszkoreit, L. Jones, A. N. Gomez, \. Kaiser, and I. Polosukhin. Attention is all you need. In Advances in neural information processing systems, pages 5998-6008, 2017.

J. Verbesselt, A. Zeileis, and M. Herold. Near real-time disturbance detection using satellite image time series. Remote Sensing of Environment, 123:98-108, Aug. 2012. ISSN 0034-4257. doi: 10.1016/j.rse.2012.02.022. URL https://www.sciencedirect.com/science/ article/pii/s0034425712001150.

A. Von Hase, M. Rouget, K. Maze, and N. Helme. A fine-scale conservation plan for Cape lowlands renosterveld: technical report. Report CCU, 2(03):104, 2003.

A. M. Wilson, A. M. Latimer, and J. A. J. Silander. Climatic controls on ecosystem resilience: Postfire regeneration in the Cape Floristic Region of South Africa. Proceedings of the National Academy of Sciences of the United States of America, 112(29):9058-9063, 2015. ISSN 10916490 (Electronic). doi: 10.1073/pnas.1416710112.

J. Yang, P. Gong, R. Fu, M. Zhang, J. Chen, S. Liang, B. Xu, J. Shi, and R. Dickinson. The role of satellite remote sensing in climate change studies. Nature climate change, 3(10):875-883, 2013.

S. Ye, J. Rogan, Z. Zhu, and J. R. Eastman. A near-real-time approach for monitoring forest disturbance using Landsat time series: stochastic continuous change detection. Remote Sensing of Environment, 252:112167, Jan. 2021a. ISSN 0034-4257. doi: 10.1016/j.rse. 2020.112167. URL https://www.sciencedirect.com/science/article/pii/ S003442572030540X.

S. Ye, J. Rogan, Z. Zhu, T. J. Hawbaker, S. J. Hart, R. A. Andrus, A. J. H. Meddens, J. A. Hicke, J. R. Eastman, and D. Kulakowski. Detecting subtle change from dense Landsat time series: Case studies of mountain pine beetle and spruce beetle disturbance. Remote Sensing of Environment, 263:112560, Sept. 2021b. ISSN 0034-4257. doi: 10.1016/j.rse. 2021.112560. URL https://www.sciencedirect.com/science/article/pii/ S0034425721002807.

Q. Zhou, J. Rover, J. Brown, B. Worstell, D. Howard, Z. Wu, A. L. Gallant, B. Rundquist, and M. Burke. Monitoring Landscape Dynamics in Central U.S. Grasslands with Harmonized Landsat-8 and Sentinel-2 Time Series Data. Remote Sensing, 11(3):328, Jan. 2019. doi: 10.3390/rs11030328. URL https://www.mdpi.com/2072-4292/11/3/328.

Z. Zhu and C. E. Woodcock. Continuous change detection and classification of land cover using all available Landsat data. Remote Sensing of Environment, 144:152-171, Mar. 2014. ISSN 0034-4257. doi: 10.1016/j.rse.2014.01.011. URL https://www.sciencedirect.com/ science/article/pii/S0034425714000248.

Z. Zhu, J. Zhang, Z. Yang, A. H. Aljaddani, W. B. Cohen, S. Qiu, and C. Zhou. Continuous monitoring of land disturbance based on Landsat time series. Remote Sensing of Environment 238:111116, Mar. 2020. ISSN 0034-4257. doi: 10.1016/j.rse.2019.03.009. URL https: //www.sciencedirect.com/science/article/pii/s0034425719301002. 